\title{
First application of a tungsten single-crystal positron source at the KEK B factory
}

\author{
T. Suwada, ${ }^{1, *}$ M. Satoh, ${ }^{1}$ K. Furukawa, ${ }^{1}$ T. Kamitani, ${ }^{1}$ T. Sugimura, ${ }^{1}$ K. Umemori, ${ }^{2}$ H. Okuno, ${ }^{3}$ Y. Endou,${ }^{4}$ T. Haruna, ${ }^{4}$ \\ R. Hamatsu, ${ }^{4}$ T. Sumiyoshi, ${ }^{4}$ K. Yoshida, ${ }^{5}$ A. P. Potylitsyn, ${ }^{6}$ I. S. Tropin, ${ }^{6}$ and R. Chehab $^{7}$ \\ ${ }^{1}$ Accelerator Laboratory, High Energy Accelerator Research Organization (KEK), 1-1 Oho, Tsukuba, Ibaraki 305-0801, Japan \\ ${ }^{2}$ Institute of Materials Structure Science, High Energy Accelerator Research Organization (KEK), 1-1 Oho, \\ Tsukuba, Ibaraki 305-0801, Japan \\ ${ }^{3}$ Institute of Particle and Nuclear Studies, High Energy Accelerator Research Organization (KEK), 1-1 Oho, \\ Tsukuba, Ibaraki 305-0801, Japan \\ ${ }^{4}$ Department of Physics, Tokyo Metropolitan University, 1-1 Minami-Ohsawa, Hachioji, Tokyo 192-0397, Japan \\ ${ }^{5}$ Kyushu Synchrotron Light Research Center, 8-7 Yayoigaoka, Tosu, Saga, 841-0005, Japan \\ ${ }^{6}$ Nuclear Physics Institute, Tomsk Polytechnic University, 634050, P.O. Box 25, Tomsk, Russia \\ ${ }^{7}$ IPNL, IN2P3-CNRS, Universite Claude Bernald 1, 69622-Villeurbanne, France
}

(Received 29 November 2006; published 10 July 2007)

\begin{abstract}
A new tungsten single-crystal target has been successfully employed at the positron source of the KEKB injector linac. The crystal thickness was determined to be $10.5 \mathrm{~mm}$ based on previous systematic measurements of the positron-production efficiency. The crystal axis, $\langle 111\rangle$, was precisely aligned to the direction of the $4-\mathrm{GeV}$ primary electron beam. The positron yield increased by $\sim 25 \%$ compared to that for a conventional tungsten plate with a thickness of $14 \mathrm{~mm}$. On the contrary, the steady-state heat load on the crystal target decreased by $\sim 20 \%$. After a two-month operation, no degradation of the positronproduction efficiency was observed, and the crystal target has been stably operating at the KEK B factory.
\end{abstract}

DOI: 10.1103/PhysRevSTAB.10.073501

\section{INTRODUCTION}

In order to achieve high luminosities in high-energy $e^{+} e^{-}$collider experiments, positron sources must be reinforced, especially towards the next generation of B factories and $e^{+} e^{-}$linear colliders.

In a conventional positron source, positrons are produced by high-energy electrons hitting a heavy-metal target, and low-momentum positrons are captured and accelerated in the succeeding positron capture section. The maximum positron-production efficiencies are obtained with an optimized target thickness of 4-5 $X_{0}$ (radiation length) and with a typical momentum acceptance of 5-25 MeV/c for 4-8 GeV incident electrons. In this case, a possible scheme to increase the positron intensity is to increase the incident electron intensity and energy. However, the allowable heat load on the target limits the beam power of the incident electrons.

One promising approach to improve the positronproduction efficiency is to use a crystal-assisted positron source first proposed by Chehab et al. [1] in 1989. When high-energy electrons hit a single crystal in the direction of the crystal axis, intense low-energy photons are produced due to channeling radiation and coherent bremsstrahlung [2]. These photons create $e^{+} e^{-}$pairs in the same crystal target. Thus, it is expected that the crystal target could increase the obtainable maximum positrons compared with the conventional heavy-metal target.

Intensive theoretical investigations [3-6] have indicated that the optimum thickness is less for the crystal target and

\footnotetext{
*tsuyoshi.suwada@kek.jp
}

PACS numbers: 29.17.+w, 29.25.-t, 41.75.Ht, 61.85.+p

that the energy deposition in the crystal target is considerably reduced compared with that of a conventional target. Also, they have shown that the crystal effect is much larger at higher incident electron energies.

Proof-of-principle experiments for tungsten crystal targets have been carried out at LAL-Orsay [7], CERN (WA103) [8,9], and KEK [10-13] in the 1-10 GeV energy region. The enhancement of photon production from the tungsten crystal in an axial channeling condition was measured at the 2-GeV Orsay linac [7]. The positron-yield enhancement at the axial channeling condition was first confirmed experimentally by Yoshida et al. with a 1.2-GeV electron beam from the electron synchrotron at the Institute for Nuclear Study, University of Tokyo [10]. Chehab et al. studied positron-yield enhancements from tungsten crystal targets either alone or in combination with conventional tungsten with 6 and $10 \mathrm{GeV}$ electrons at CERN-SPS [9]. A test experiment of the crystal target was made at the KEKB injector linac in 1999 by using a composite target made of a 1.7-mm-thick tungsten crystal and a 7.0-mm-thick conventional tungsten plate. The results show that the positron yield was enhanced by $40 \%$ compared with that from a disoriented target with 3-GeV electrons [11].

Encouraged by these stimulating results, we proceeded to systematic studies on the positron-production efficiencies with tungsten crystals having various thicknesses using 4- and $8-\mathrm{GeV}$ electron beams $[12,13]$. Based on these results, we optimized the thickness of the tungsten crystal at a $4-\mathrm{GeV}$ primary electron beam. After developing a crystal-axis alignment technique, we installed a tungsten crystal target at the positron source of the KEKB injector linac. In this report, the characteristics and operational 
performance of the tungsten crystal target are described in detail.

\section{POSITRON SOURCE OF THE KEKB INJECTOR LINAC}

The KEK B factory (KEKB) [14] has been progressing in order to test $C P$ violation in the decay of $\mathrm{B}$ mesons since 1999. KEKB is an asymmetric electron-positron collider comprising $3.5-\mathrm{GeV}$ positron and $8-\mathrm{GeV}$ electron storage rings. The KEKB injector linac [15] injects single-bunch positron and electron beams directly into the KEKB rings. The beam charges were designed to be $0.64 \mathrm{nC} /$ bunch and $1.3 \mathrm{nC} /$ bunch at the end of the injector linac with a maximum repetition rate of $50 \mathrm{~Hz}$ for positron and electron beams, respectively. High-current primary electron beams ( $\sim 10 \mathrm{nC} /$ bunch) are required in order to generate a sufficient number of positrons. Since the KEKB is a factory machine, a well-controlled operation of the injector linac is required for maximizing the injection rates of the electron and positron beams in order to increase the integrated luminosity. For this purpose, two schemes were developed for the positron beams of the injector linac. One is a simultaneous acceleration and injection scheme of two bunches separated by $96 \mathrm{~ns}$ in an rf pulse [16]. The twobunch injection scheme has successfully enabled us to increase the injection rate of the positron beam by a factor of 2 . The other is to apply a tungsten crystal target in order to further increase the positron intensity.

Figure 1 shows a schematic drawing of the positron source of the KEKB injector linac. The positron source comprises a positron-production target and a positron capture section. The positrons are generated by the $4-\mathrm{GeV}$ primary electron beam impinging on the tungsten target. The average beam power is $2 \mathrm{~kW}$ at a maximum repetition rate of $50 \mathrm{~Hz}$. The typical transverse beam size is $0.7 \mathrm{~mm}$ (rms) in radius, and the typical horizontal and vertical normalized emittances are 660 and $360 \mathrm{~mm} \mathrm{rad}(\mathrm{rms})$ on average at the target. The horizontal (vertical) angular spread at the target is estimated to be $0.2(0.1) \mathrm{mrad}$ (rms). These angular spreads need to be controlled within the critical angle for axial channeling [2], which is $0.61 \mathrm{mrad}$ at $4 \mathrm{GeV}$ in a tungsten crystal.
The previously used 14-mm-thick $\left(4 X_{0}\right)$ conventional tungsten target was replaced by a 10.5 -mm-thick tungsten crystal in September 2006 without any significant modification of the accelerator layout.

The positron capture section $[15,17]$ comprises a 45 mm-long pulse solenoid with a field strength of $2 \mathrm{~T}$, an 8$\mathrm{m}$-long DC solenoid with a field strength of $0.4 \mathrm{~T}$, and two 1-m-long acceleration and two 2-m-long acceleration structures, which are installed inside the DC solenoid. The positrons generated from the target are captured by these two types of solenoidal magnetic fields (so-called quarter-wave transformer) and accelerated by the succeeding accelerator sections. The electrons generated along with the positrons are stopped by a positron/electron separator (chicane) comprised of four rectangular magnets and a beam stopper at the center of the chicane. The geometrical acceptance of the capture section is about $420 \mathrm{~mm} \mathrm{rad}$ and the typical momentum acceptance is about $24 \%$ at the momentum of $10 \mathrm{MeV} / c$, which are based on simulations carried out with conventional tungsten plates. Further details of the design of the positron source are described elsewhere [17].

\section{TUNGSTEN CRYSTAL TARGET}

\section{A. Crystal preparation}

Tungsten single crystals with several thicknesses were fabricated at collaborating Tomsk Polytechnic University. They were developed based on the purification of an initial tungsten sample and a solid-state recrystallization process of large-size crystal ingots. Also, a technique for sample cutting along the crystallographic planes without any crystal quality damage was developed. The surface mosaic spreads of the crystal structure on both the beam-entrance and the beam-exit sides were measured by an x-ray scattering method within $0.5 \mathrm{mrad}$. The consistency of the crystal axis on both sides after assembling the tungsten crystal into the copper body was also measured by test experiments using the $4-\mathrm{GeV}$ electron beam.

\section{B. Optimization of the target thickness}

For practical applications of the crystal target at the KEKB injector linac, we systematically studied the

\section{- Primary electron beam \\ $\rightarrow$ Electron beam \\ $\longrightarrow$ Positron beam}

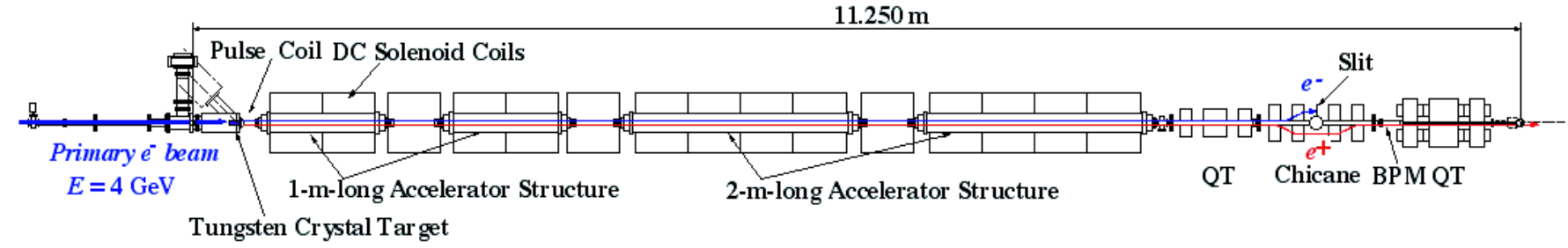

FIG. 1. (Color) Layout of the positron source at the KEKB injector linac. QT, quadrupole triplet. 


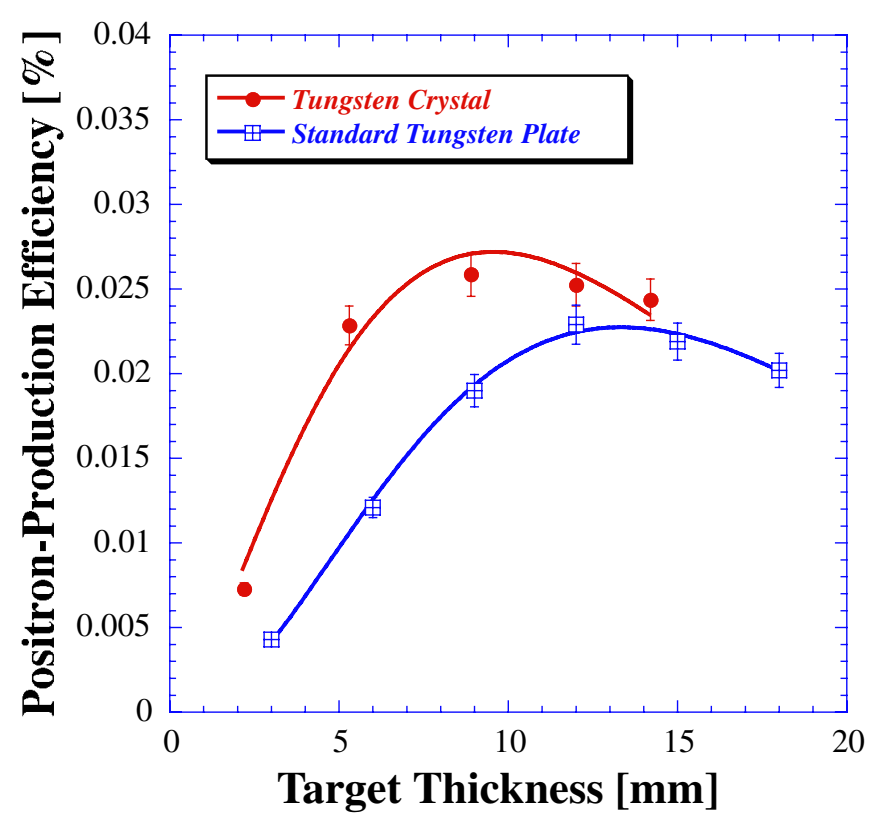

FIG. 2. (Color) Positron-production efficiencies measured for the tungsten crystal as a function of the crystal thickness (see [13] in detail). The incident electron energy and positron momentum were $4 \mathrm{GeV}$ and $20 \mathrm{MeV} / c$, respectively. The solid curves through the data are gamma-function fits of the data.

positron-production efficiency as a function of the crystal thickness at an incident electron energy of $4 \mathrm{GeV}$ [13]. The positrons produced in the forward direction were measured by a magnetic spectrometer with an angular acceptance of about $1 \mathrm{msr}$ and a typical momentum acceptance of $2.4 \%$ (at the momentum of $20 \mathrm{MeV} / c$ ) in the momentum range of $5-20 \mathrm{MeV} / c$. Tungsten crystal targets with various thicknesses were tested and the optimum thickness was investigated with an incident electron energy of $4 \mathrm{GeV}$. Figure 2 shows the result of the positron-production efficiency measured at a momentum of $20 \mathrm{MeV} / c$. The optimum thickness giving the maximum positron-production efficiency was obtained to be about $10 \mathrm{~mm}$. As a comparison, it was about $14 \mathrm{~mm}$ for the conventional tungsten plate. From these data, it was concluded that the maximum positron yield for the 10-mm-thick tungsten crystal target was about $26 \%$ larger than that for the 14-mm-thick conventional tungsten plate at an incident electron energy of $4 \mathrm{GeV}$ and a momentum of $20 \mathrm{MeV} / c$. The peak width of the rocking curve was also obtained to be $40 \mathrm{mrad}$ in FWHM (see [13]). The relatively large peak width helps us to relax the tolerance of the target axis alignment with respect to the direction of the primary electron beam.

\section{Target fabrication}

Figure 3(a) shows a mechanical drawing of the target assembly with a tungsten crystal target. The crystal target with a thickness of $10.5 \mathrm{~mm}$ and a cross section of $5 \mathrm{~mm} \times$ $5 \mathrm{~mm}$ is fixed at the center of a cylindrical copper body for (a)

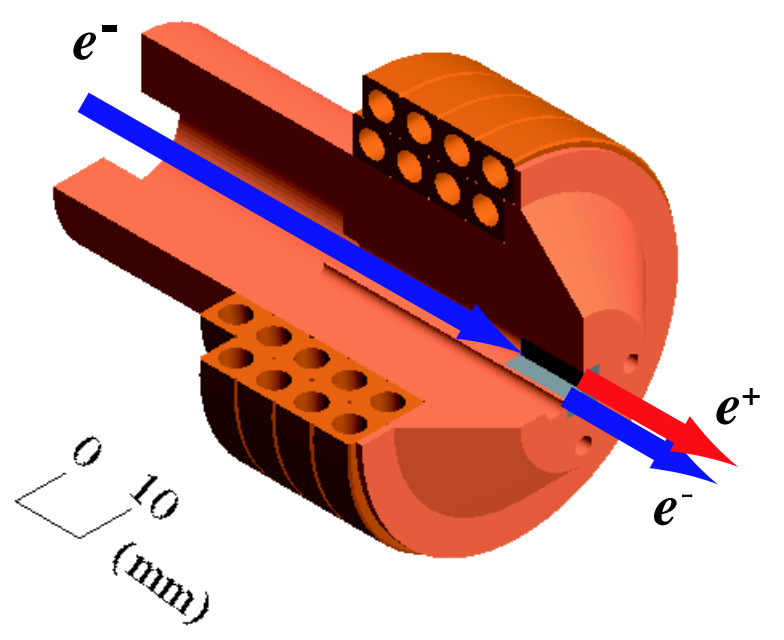

(b)

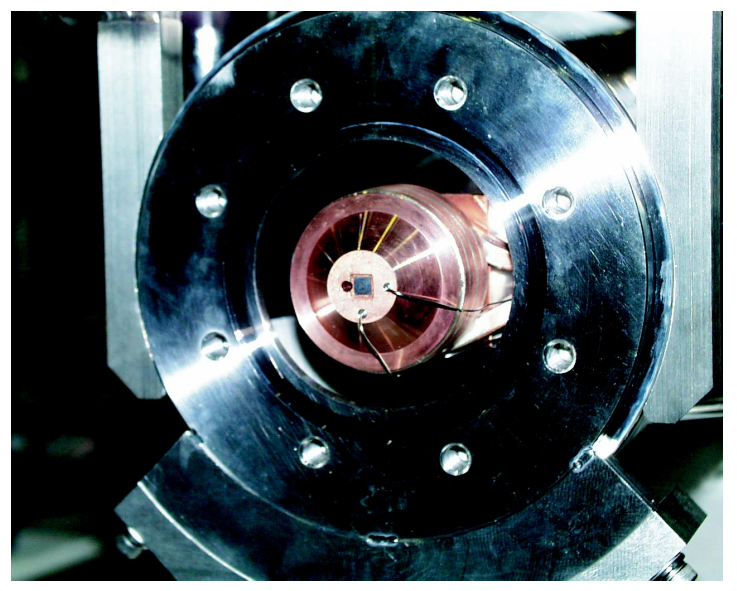

FIG. 3. (Color) (a) Mechanical drawing of the target assembly. The tungsten crystal is fixed to the center of a cylindrical copper body with a diameter of $50 \mathrm{~mm}$ for water cooling with a hot isostatic pressing technique. The heat on the crystal target is conducted through a cooling water channel (4 mm diameter) composed of a copper pipe. Primary electrons (blue arrow) impinge the tungsten crystal target and are converted to electrons (blue arrow) and positrons (red arrow). (b) Target assembly installed in a vacuum chamber seen from downstream. Two thermocouples are mounted $7.5 \mathrm{~mm}$ away from the center of the tungsten crystal, and a small hole with a $3-\mathrm{mm}$ diameter is penetrated through the copper body for transport of the electron beam.

water cooling with a hot isostatic pressing technique. On the other hand, a cylindrical rod with a thickness of $14 \mathrm{~mm}$ and a diameter of $5 \mathrm{~mm}$ for the previously used tungsten target was fixed at the center of a similar cylindrical copper body. The geometrical structure of the copper body is exactly the same as that of the crystal tungsten target assembly except only for the difference of the geometrical shape of the tungsten target. Figure 3(b) also shows the target assembly installed in a vacuum chamber. The heat at the target is conducted through a cooling water channel wound around a copper body of 50-mm diameter, and it is 
removed by the cooling water with a flow speed of $1.5 \mathrm{l} / \mathrm{min}$ and the base temperature of $30^{\circ} \mathrm{C}$ at the inlet. Two thermocouples are mounted near the tungsten crystal in order to measure the temperature rise. The target assembly linked with a mechanical linear actuator is mounted in a vacuum chamber. It is inserted into the beam line by a linear actuator for an injection request of the positron beam.

Since there was no room to install a goniometer for controlling the crystal axis with respect to the incident electron beam, the target assembly was carefully fabricated so that the central axis of the cylindrical copper body corresponded exactly to the crystal axis, $\langle 111\rangle$. In order to precisely align these two axes, the relative inclination angles between them were obtained by setting the copper body on a positioning jig and by measuring the crystal axis with an x-ray Bragg reflection method. Based on the results, the copper body was mechanically remachined so as to correct for any misalignment. After this procedure, the inclination angles were measured again and the results showed that the two axes were in agreement within an accuracy of $\pm 1 \mathrm{mrad}$.

\section{Alignment of the target assembly}

After the mounting of the target assembly into the vacuum chamber, the final alignment of the target assembly to the incident direction of the primary electron beam was carefully made by using a precisely aligned telescope with an angular resolution of $0.5 \mathrm{mrad}$. After these mechanical alignment procedures, the positron chamber was installed at the positron source. The alignment accuracy of the target assembly in terms of the direction of the crystal axis was estimated to be within \pm 3 mrad in total. From the rocking-curve peak width of the positron yields, the reduction of the positron yield due to the misalignment was estimated to be only $\sim 1 \%$.

\section{PERFORMANCE OF THE CRYSTAL TARGET}

\section{A. Positron-production efficiency}

Beam tests were carried out by adjusting the incident angles of the primary electron beam at the crystal target with two sets (horizontal and vertical) of upstream steering magnets. The beam charges of the primary electrons and positrons were measured with stripline-type beam-position monitors (BPMs) [18] installed both upstream and downstream of the positron source. The BPMs were directly calibrated based on single-bunch beam-charge measurements with a Faraday cup [19]. The charges of the primary electron beam was $7.5 \mathrm{nC} /$ bunch on average during the beam tests. The typical energy spread and the bunch length were $2.6 \%$ at $1.7 \mathrm{GeV}$ (defined by an occupation of $90 \%$ electrons) and 13 ps (FWHM), respectively. The incident angles were controlled within angular ranges of $\pm 2 \mathrm{mrad}$, since the controlled ranges of the incident angles were

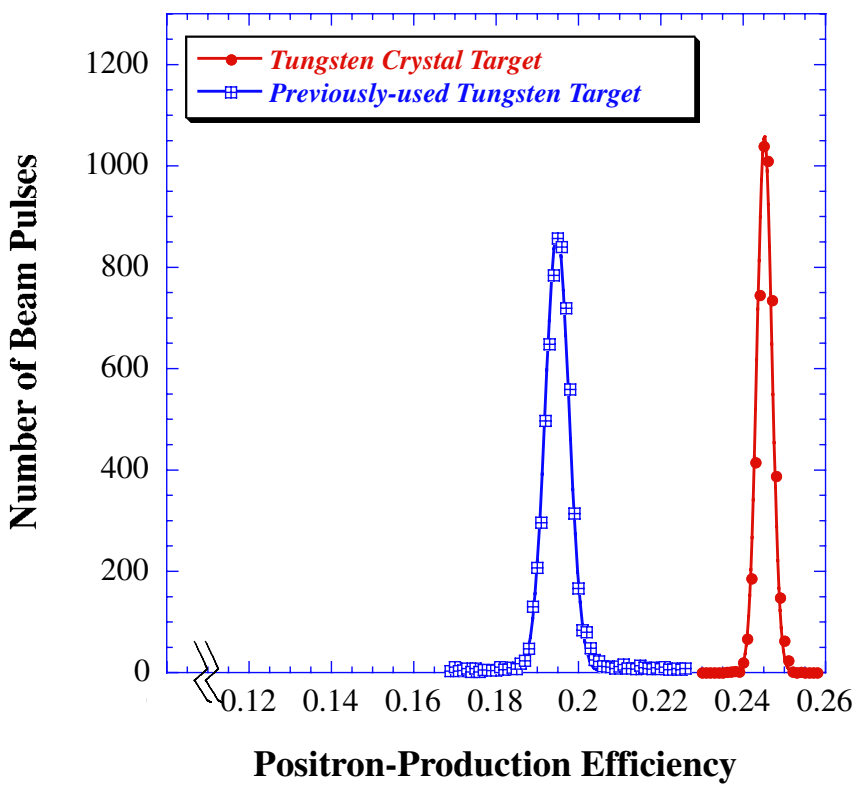

FIG. 4. (Color) Positron-production efficiencies of the 1st bunch measured for each beam pulse. For the sake of comparison, the data of the previous tungsten target are plotted. The solid lines are Gaussian-function fits of the data.

limited due to a lack of sufficient field strengths of the steering magnets.

After optimization of the two sets of steering magnets, both the positron and primary electron charges were simultaneously measured with the upstream and downstream BPMs. Figure 4 shows the distributions of the positronproduction efficiencies $\left(N_{e^{+}} / N_{e^{-}}\right)$of the 1st bunch measured for each beam pulse, where $N_{e^{+}}$is the number of positrons captured in the positron capture section and $N_{e^{-}}$ is the number of the primary electrons. For the sake of comparison, the data with the previously used conventional tungsten target obtained in June 2006 are also shown in this figure. The results show that the positron-production efficiencies of the first (second) bunch are $0.25 \pm 0.01(0.26 \pm$ $0.01)$ and $0.2 \pm 0.01(0.2 \pm 0.01)$ on average for the tungsten crystal target and the previously used tungsten target, respectively. The increase in the positrons for the 1st (2nd) bunch from the crystal tungsten is $25 \% \pm 2 \%(28 \% \pm 2 \%)$ on average, where the errors indicate one standarddeviation uncertainties. The positron-production efficiencies of both bunches are consistent with each other within the experimental errors. These results are quantitatively in agreement with our previous results obtained by experimental studies described before.

\section{B. Operational performance}

Figure 5 shows time traces of the positron-production efficiency of the two bunches averaged every five days after the start-up of KEKB operation in September. The results show that the positron-production efficiencies of the 


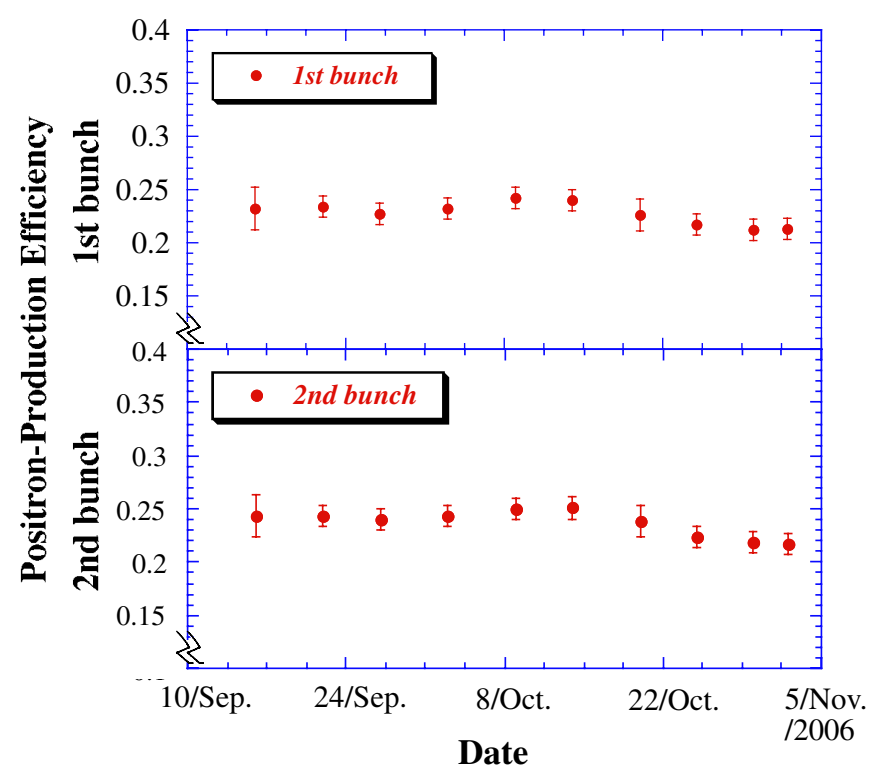

FIG. 5. (Color) Time traces of the positron-production efficiency of the 1 st and 2 nd bunches averaged every five days.

two bunches have been stably kept during the two-month operation.

The horizontal and vertical normalized emittances of the positron beam were also measured to be $1440 \pm$ $40 \mathrm{~mm} \mathrm{rad}(1500 \pm 80 \mathrm{~mm} \mathrm{rad})$ and $1640 \pm 110 \mathrm{~mm} \mathrm{rad}$ $(1740 \pm 60 \mathrm{~mm} \mathrm{rad})$ for the first (second) bunch on average with downstream wire scanners, respectively. The results are consistent with those obtained for the previously used tungsten target. This means that the transverse emittances are mainly determined by the acceptance of the positron capture section.

The present results were obtained at instantaneous charges of $7.7 \mathrm{nC} /$ bunch on average and the primary electron beam energy of $4 \mathrm{GeV}$. We previously checked the intensity dependence of the positron-yield enhancement from a $2.55-\mathrm{mm}$-thick silicon crystal target for $8-\mathrm{GeV}$ primary electron beams up to $1.9 \mathrm{nC} /$ bunch [20]. No degradation was observed in this test experiment. The present results also show that the positron yields increase linearly with an increase in the primary electron intensity without any abnormal behavior, as shown in Fig. 6. This means that the crystal effect is not affected at least up to this intensity.

Steady-state heat loads of the crystal target were also obtained by measuring the temperature rises as a function of the beam repetition rate with two thermocouples mounted inside the copper body. The temperature rise is defined by the average temperature of the two thermocouples. The temperature rise was $\Delta T \approx 13.2^{\circ} \mathrm{C}$ at a beam repetition rate of $50 \mathrm{~Hz}$ under one-bunch operation with a bunch charge of $7.8 \mathrm{nC}$, on average, where the similar conditions described in Sec. IIIC were applied to the cooling water during these measurements. Figure 7 shows the variations in the temperature rise of the crystal target

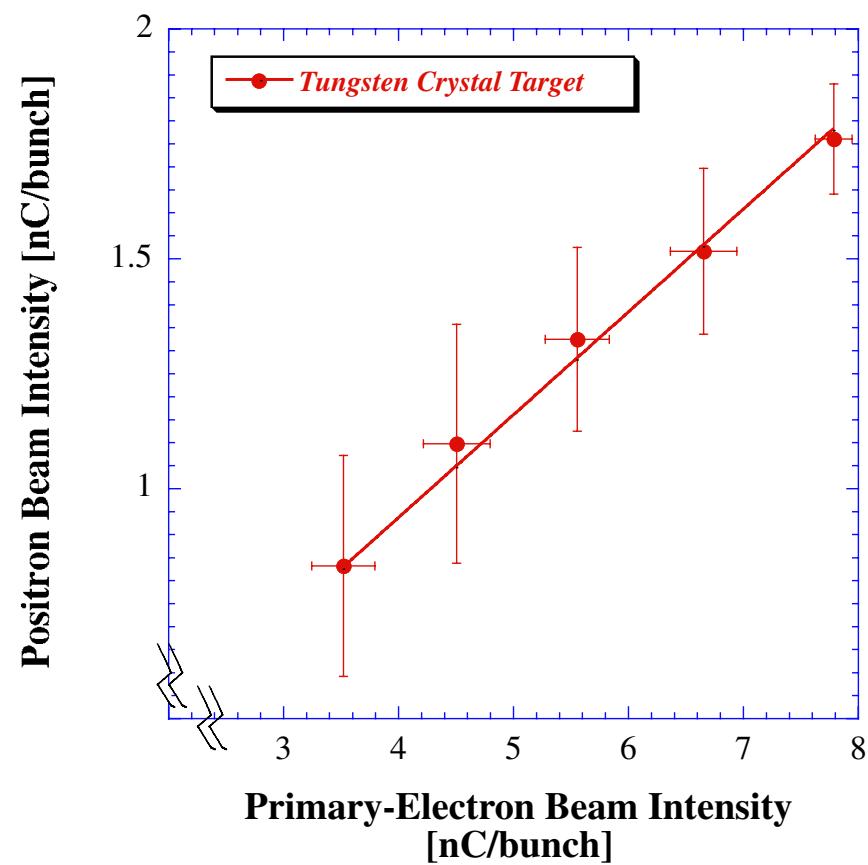

FIG. 6. (Color) Positron beam intensity plotted versus the primary electron beam intensity in a bunch. The solid line through the data indicates a linear-function fit of the data.

normalized by the charges of the primary electron beam as a function of the beam repetition rate. The data of the previously used tungsten target were also obtained under the exactly same conditions applied to the cooling water.

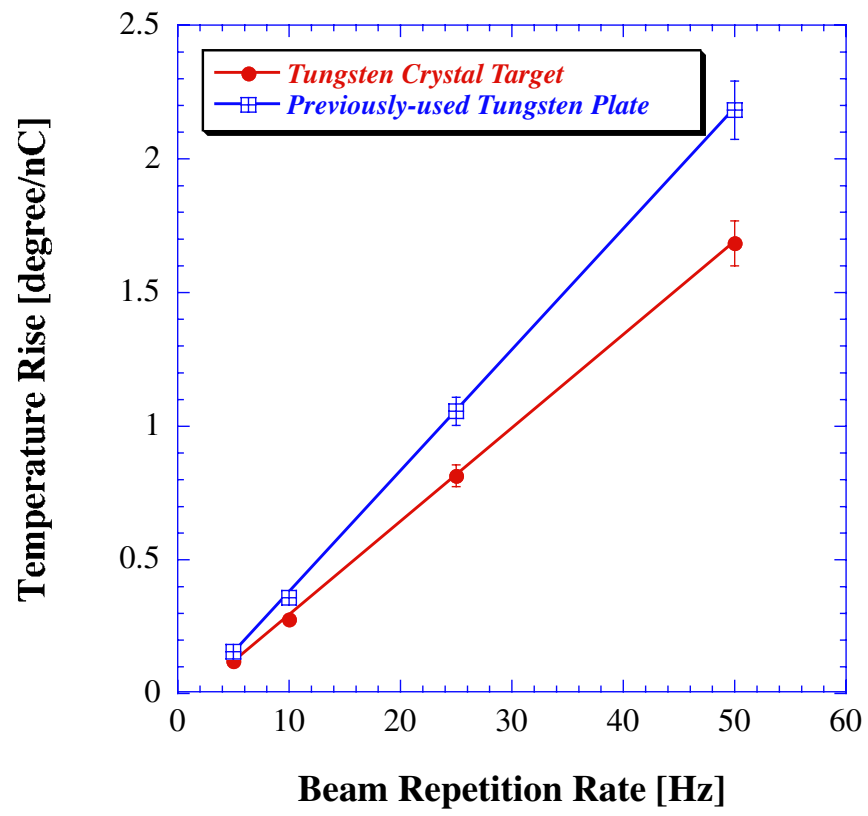

FIG. 7. (Color) Variations of the temperature rise of the tungsten crystal target as a function of the beam repetition rate under onebunch operation. In this figure the temperature rise is normalized by the average beam charges of the primary electron beam. For the sake of comparison, the data of the previously used tungsten target are plotted. 
These results confirm that the steady-state heat load on the crystal target as a function of the beam repetition rate is clearly reduced by about $20 \%$ compared with that of the previously used tungsten target.

The power densities deposited on the crystal and conventional targets have been investigated theoretically by Artru et al. [6] at an incident electron energy of 2-10 GeV. Based on their results, the total energy deposit on a $10-\mathrm{mm}-$ thick crystal is estimated to be $\sim 20 \%$ lower than that of a 14-mm-thick conventional tungsten at an incident electron energy of $4 \mathrm{GeV}$. The present result is consistent with their theoretical estimations.

The new positron source has been stably operating without any significant reduction of the positron-production efficiency during these two months. For long-term KEKB operation, it would be useful to apply a feedback control to the incident angles of the primary electron beam with two successive BPMs in order to keep the positron-production efficiency as high as possible.

The integrated electron flux hitting the crystal target has amounted to about $5.5 \times 10^{7} \mathrm{nC} / \mathrm{mm}^{2}$. As for the radiation damage effect, Artru et al. [21] tested on a $0.3-\mathrm{mm}$ thick tungsten crystal placed in front of the positron converter of the SLAC Linear Collider up to an integrated electron flux of $3.2 \times 10^{8} \mathrm{nC} / \mathrm{mm}^{2}$. No damage to the crystal structure was found after irradiation. By accepting these numbers, we expect that the present tungsten crystal target will be used for one year without serious radiation damage. However, clear answers will be obtained in future operational experience obtained at the KEKB injector linac.

\section{CONCLUSIONS}

We have successfully applied a new tungsten crystal target for generating an intense positron beam at the positron source of the KEKB injector linac. The positron intensity increased by $\sim 25 \%$ compared with that from the previously used conventional tungsten target, and on the contrary, the steady-state heat load on the crystal target was reduced by $\sim 20 \%$. It has boosted the positron intensity to its maximum since the beginning of the KEKB operation in 1999. This is the first application of a crystal target to high-energy electron/positron linacs. Useful information concerning radiation damage and stability of the crystal target will be obtained through KEKB operation. The present result encourages us to consider the application of crystal targets in the next generation of B factories and $e^{+} e^{-}$linear colliders.

\section{ACKNOWLEDGMENTS}

The authors would like to thank Professor A. Enomoto and Professor K. Oide of Accelerator Laboratory of KEK for their continuous support on this work. They also thank Professor H. Kawata and Mr. M. Satoh of Photon Factory of KEK, who helped to conduct the crystal-axis measurements. This work was supported by the collaborative R\&D program at KEK, by the Grant-in-Aid of Ministry of Education, Culture, Sports, Science and Technology (MEXT) of Japan (Grant No. 17540377), and by the grant of Ministry of Education and Science of the Russian Federation "Advancement of the Scientific Potential of High Education" (Grant No. 2.1.1.889).

[1] R. Chehab, F. Couchot, A. R. Nyaiesh, F. Richard, and X. Artru, Proceedings of the 1989 IEEE Particle Accelerator Conference (PAC'89), Chicago, IL, USA, 1989, p. 283.

[2] V.N. Baier, V.M. Katkov, and V.M. Strakhovenko, Electromagnetic Processes at High Energies in Oriented Single Crystals (World Scientific, Singapore, 1998).

[3] X. Artru, Nucl. Instrum. Methods Phys. Res., Sect. B 48, 278 (1990).

[4] V. N. Baier, V. M. Katkov, and V. M. Strakhovenko, Nucl. Instrum. Methods Phys. Res., Sect. B 103, 147 (1995).

[5] V. N. Baier and V. M. Strakhovenko, Phys. Rev. ST Accel. Beams 5, 121001 (2002).

[6] X. Artru, R. Chehab, M. Chevallier, and V. Strakhovenko, Phys. Rev. ST Accel. Beams 6, 091003 (2003).

[7] X. Artru et al., Nucl. Instrum. Methods Phys. Res., Sect. B 119, 246 (1996).

[8] R. Chehab et al., Phys. Lett. B 525, 41 (2002).

[9] X. Artru et al., Nucl. Instrum. Methods Phys. Res., Sect. B 240, 762 (2005).

[10] K. Yoshida et al., Phys. Rev. Lett. 80, 1437 (1998).

[11] M. Inoue et al., Nucl. Instrum. Methods Phys. Res., Sect. B 173, 104 (2001).

[12] T. Suwada et al., Phys. Rev. E 67, 016502 (2003).

[13] T. Suwada et al., in the 2006 International Linear Accelerator Conference (LINAC'06), Knoxville Convention Center, TN, USA, 2006.

[14] K. Akai et al., Nucl. Instrum. Methods Phys. Res., Sect. A 499, 191 (2003).

[15] I. Abe et al., Nucl. Instrum. Methods Phys. Res., Sect. A 499, 167 (2003).

[16] K. Furukawa, N. Kamikubota, T. Suwada, and T. Obata, Proceedings International Conference Accelerator and Large Experimental Physics Control Systems (ICALEPCS2001), San Jose, USA, 2001 (SLAC Report No. SLAC-R-592, eConfC011127), p. 266.

[17] A. Enomoto et al., Proceedings of the Third European Particle Accelerator Conference (EPAC'92), Berlin, Germany, 1992, Vol. 1, p. 524.

[18] T. Suwada, N. Kamikubota, H. Fukuma, N. Akasaka, and H. Kobayashi, Nucl. Instrum. Methods Phys. Res., Sect. A 440, 307 (2000).

[19] T. Suwada, S. Ohsawa, K. Furukawa, and N. Akasaka, Jpn. J. Appl. Phys. 39, 628 (2000).

[20] T. Suwada et al., Nucl. Instrum. Methods Phys. Res., Sect. B 252, 142 (2006).

[21] X. Artru et al., Proceedings of the Sixth European Particle Accelerator Conference (EPAC'98), Stockholm, Sweden, 1998, Vol. 2, p. 1394. 